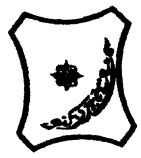

Bayero Journal of Pure and Applied Sciences, 10(1): 32 - 38

Received: December, 2016

Accepted: March, 2017

ISSN $2006-6996$

\title{
THE BENEFICIAL ROLE OF RESVERATROL ON CHLORPYRIFOS-INDUCED COGNITIVE IMPAIRMENT AND LIPID PEROXIDATION BIOMARKER IN SWISS ALBINO MICE
}

\author{
${ }^{1}$ Adamu, B.Y., ${ }^{2}$ Alhassan, A.W., ${ }^{2}$ Saleh, ${ }^{2}$ M.I.A., ${ }^{2}$ Aliyu, M., ${ }^{2}$ Iliya, B.T., ${ }^{2}$ Zuberu, J., ${ }^{2}$ Yusuf, \\ A., ${ }^{1}$ Garkuwa, U.A. and ${ }^{3}$ Lawan, $I$.
}

1. Department of Human Physiology, Faculty of Basic Medical Sciences, Bauchi State University Gadau, Bauchi State, Nigeria.

2. Department of Human Physiology, Faculty of Medicine, Ahmadu Bello University Zaria, Kaduna State, Nigeria.

3. Department of Clinical Pharmacy, Faculty of Pharmaceutical Sciences, Ahmadu Bello University Zaria, Kaduna State, Nigeria.

\begin{abstract}
Chlorpyrifos (CPF) has been associated with cognitive and psychomotor impairments in both humans and animals. This cognitive impairment has been linked to its enhanced reactive oxygen species (ROS) generating capacity. Therefore, antioxidant treatment may provide a novel therapeutic window for the management of these related impairments. The aim of this work was to evaluate the beneficial role of resveratrol on chlorpyrifos-induced cognitive impairment and lipid peroxidation biomarker in Swiss albino mice. Swiss albino mice were divided into (6) six groups of five each ( $n=5)$. Group I served as the control and were administered olive oil (2 ml/ $\mathrm{kg})$, group II received carboxymethylcellulose (CMC) $10 \mathrm{mg} / \mathrm{kg}$, group III received resveratrol $30 \mathrm{mg} / \mathrm{kg}$, group IV received chlorpyrifos (CPF) $3 \mathrm{mg} / \mathrm{kg}$, group $V$ received CPF (3 $\mathrm{mg} / \mathrm{kg}$ ) after the oral administration of resveratrol (30 mg/kg) and group VI received Vitamin E (Vit E) $100 \mathrm{mg} / \mathrm{kg} \mathrm{All}$ administrations were done by oral gavage for a duration of 21 days. Cognitive function was assessed using $Y$-maze, and Novel object recognition tests and oxidative stress was evaluated using oxidative biomarkers techniques. The results obtained showed that resveratrol at dose $30 \mathrm{mg} / \mathrm{kg}$ significantly $(p<0.05)$ improved cognitive impairment and significantly decreased $(p<0.05)$ malondialdehyde (MDA) concentration when compared with the control. In conclusion, $30 \mathrm{mg} / \mathrm{kg}$ resveratrol suppressed memory impairment, decreased malondialdehyde levels, increased catalase activity, superoxide dismutase activity and glutathione levels in our chlorpyrifos-induced cognitive impairment mice model.
\end{abstract}

Keywords: cognition, chlorpyrifos, resveratrol, memory

\section{INTRODUCTION}

There has been a global increase in pesticides usage due to the compelling need to feed the everincreasing human and animal populations, and to reduce the incidence of food and vector-borne diseases (Ambali, et al., 2012). These health and economic benefits of pesticide usage are achieved not without simultaneous potential health risks and adverse health outcomes in non-target species, including man (Abdollahi et al., 2004).

Organophosphate (OP) compounds are one of the most widely used pesticides accounting for about $50 \%$ of the global pesticide use (Ambali, et al., 2012). This may be of particular concern given the widespread use of OP pesticide in household, agricultural, and commercial environments worldwide (Terry et al., 2007). Human studies in agricultural communities in developing countries have shown that cumulative exposure to OP is associated with cognitive and psychomotor impairments (Kamel and Hoppin, 2004; Kamel et al., 2007).

Chlorpyrifos (CPF) is a chlorinated OP insecticide that has enjoyed widespread use in agricultural and domestic pest control (Steenland et al., 2000; Ambali et al., 2009). It is of public health importance as CPF residues have been detected in poultry egg, meat, cow milk and milk products (Rawat et al., 2003). CPF is thought to cause oxidative stress in both humans and animals via the formation of free radicals (Terry et al., 2007). Exposure to CPF at doses that did not result in overt clinical symptoms has been reported among pesticide applicators and other farm workers (Farahat et al., 2010) thereby constituting an important source of occupational hazards to these groups of individuals.

Resveratrol (3, 5, 4'-trihydroxystilbene) is a polyphenol that occurs naturally in foods and drinks made from grapes and peanuts, and also in a number of herbal remedies, both alone and as part of plant extracts (Villaflores, et al., 2012). Resveratrol was first isolated from the roots of white hellebore (Veratrum grandiflorum O. Loes) in 1940 (Takaoka, 1940) and later, in 1963, from the roots of Polygonum cuspidatum, a plant used in traditional Chinese and Japanese medicine. Initially characterized as a phytoalexin (Nonomura et al., 1963), 
resveratrol is considered to be one of such compounds that are produced in plants during times of environmental stress of pathogenic attack (Dercks, and Creasy, 1989) or exposure to ultraviolent light (Fremont, 2000).

Cognition in humans is the ability to consciously carry out functions utilizing the human brain and includes: visual perception, selective attention, logical reasoning, construction (sentences for example) calculation, attention information processing), planning, problem-solving, comprehension and memory (Warburton, 1995).

For nearly a century, studies of cognition have shown that certain exogenous compounds can improve different types of memory and cognitive functioning in both normal and abnormal animals. However, the cognition-enhancing effects of other agents are only observed in subjects exhibiting poor baseline performance or experimentally induced deficits, whereas providing no measurable effects in normal animals (Kimet al., 2007). The aim of this study was to evaluate the effect of resveratrol on chlorpyrifosinduced cognitive deficit in Swiss Albino mice. The objectives are to determine the effect of CPF on cognitive function in mice, to determine the effect of resveratrol on CPF-induced cognitive dysfunction in mice and to determine the effect of resveratrol on CPF-induced elevation of oxidative stress biomarker: mlondialdehyde (MDA).

\section{MATERIALS AND METHODS \\ Experimental Animals}

Thirty (30) adult Swiss albino mice weighing between $25 \mathrm{~g}$ and $30 \mathrm{~g}$ were used for the study. They were fed on standard animal feed, while water was provided ad libitum. The experiment was conducted in accordance with the National Institutes of Health Guide for Care and Use of Laboratory Animals with ethical approval from the Ethical Committee of the Department of Human Physiology, Ahmadu Bello University Zaria, constituted under the Committee for the Purpose of Control and Supervision of Experiments on Animals.

\section{Chemicals}

Commercial grade CPF (25\% EC, Termicot $\AA$, Sabero Organics, G limited, Lebanon), were prepared by reconstituting in soya oil (Grand Cereals and Oil Mills Ltd., Jos, Nigeria) to make $10 \%$ stock solution and Resveratrol of analytical grade ( SIGMA Chemical Company Ltd. St. Louis USA), was dissolved in carboxymethylcellulose ( SIGMA Chemical Company Ltd. St. Louis USA) to obtain $30 \mathrm{mg} / \mathrm{kg}$ solution.

\section{Animal Treatment Schedule}

The mice were weighed and then assigned at random into 6 groups of 5 each $(n=5)$.

Group I: Olive oil $2 \mathrm{ml} / \mathrm{kg}$ (vehicle for chlorpyrifos dilution)

Group II: CMC $2 \mathrm{ml} / \mathrm{kg}$ (vehicle for resveratrol dissolution)

Group III: CPF $3 \mathrm{mg} / \mathrm{kg}$ ( $\mathrm{LD}_{50}$ of $85 \mathrm{mg} / \mathrm{kg}$ (Ambali, 2009))

Group IV: RSV 30 mg/kg b. w.

Group V: VIT E+CPF: Mice were pre-treated with Vit. $E(100 \mathrm{mg} / \mathrm{kg})$ and $3 \mathrm{mg} / \mathrm{kg}$ of CPF was administered 30 min later.
Group VI: RSV+CPF: Mice were pre-treated with resveratrol $(30 \mathrm{mg} / \mathrm{kg}$ ) and $3 \mathrm{mg} / \mathrm{kg}$ of $\mathrm{CPF}$ was administered 30 min later.

Administration was done orally for the period of 21 days at 10:00 hour daily.

\section{Cognition Tests}

\section{Novel Object Recognition Test}

The Novel object recognition test is an open field assessment of the natural tendency of rodents to investigate a novel object instead of a familiar one as well as their innate tendency to re-start exploring when they are presented with a novel environment or familiar object relocation. The choice to explore the novel object as well as the reactivation of exploration after object displacement reflects the use of learning and (recognition) memory processes (Carlini, 2011).

This task comprised two sample phases and one test trial. In each sample phase, the mice were placed and allowed to explore two copies of an identical object for a total of $5 \mathrm{~min}$. Different objects (rubber toys) were used for sample phases 1 and 2, with a delay between the sample phases of $1 \mathrm{~h}$. The test trials ( 5 min) were given $3 \mathrm{~h}$ after sample phase 2 . During the test trial, a third copy of the objects from sample phase 1 and a third copy of the objects from sample phase 2 were used. The positions of the objects in the test and the objects used in sample phase 1 and sample phase 2 were counterbalanced between the animals. If learning and recognition memory is intact, the rat will spend more time exploring the object from sample 1 (i.e., the object presented less recently) compared with the object from sample 2 (i.e., the "new" object). The recognition ratio were calculated as the difference in time spent by each animal exploring the object from sample phase 1 compared with the object from sample phase 2 divided by the total time spent exploring both objects in the first minute of the test period (Barker et al., 2007; Baxter, 2010; Gaskin, 2010; Antunes and Biala, 2012).

\section{Y-Maze Model (spatial memory test)}

The mice's Y-maze is composed of three equally spaced arms (at $120^{\circ}$, arm's length $50 \mathrm{~cm}$, width 10 $\mathrm{cm}$, and wall height $20 \mathrm{~cm}$ ). The floor of each arm is made of Perspex. Y-maze is a quick and useful initial test for general cognitive function. This test is based on the innate preference of animals to explore an arm that has not been previously explored. Y-maze function is sensitive to damage in areas concerned with learning and memory functions such as the hippocampus, and is also disrupted by drugs that cause memory loss (Hughes, 2004). The Spontaneous alternation version of $\mathrm{Y}$-maze testing was employed for the study. In this version each mouse was placed in the Y-maze for $5 \mathrm{~min}$ and the number of arms entered as well as the sequence of entries were recorded and a score was calculated to determine alternation rate. An alternation is defined as entry into all three arms consecutively (Hughes, 2004), for instance if the animal makes the following arm entries; $A, C, C . A, B, C, A, C, B, A, B, C, A$ in this example, the animal made 13 arm entries 7 of which are correct alternations. 
Bajopas Volume 10 Number 1 June, 2017

The number of maximum spontaneous alternations is then the total number of arms entered minus two, and the percentage alternation is calculated as (actual alternations /maximum alternations) $x$ 100. A high alternation rate is indicative of sustained spatial working memory as the animals must remember which arm was entered last to not re-enter it (Hughes, 2004).

$\begin{array}{llrr}\text { Estimation of } & \text { Lipid } & \text { Peroxidation } \\ \text { (malondialdehyde): } & & \\ \text { Lipid peroxidation } & \text { was } & \text { estimated }\end{array}$ spectrophotometrically as thiobarbituric acid reactive substances (TBARS). A principal component of TBARS is malondialdehyde (MDA), a product of lipid peroxidation.The assay for malondialdehyde was carried out according to the principle of colorimetry as described by Janero 1990.The assay employed the competitive inhibition enzyme immunoassay technique. A monoclonal antibody specific for MDA was pre-coated onto a microplate. A competitive inhibition reaction was launched between biotin labelled MDA and unlabelled MDA/samples (brain homogenate) with the pre-coated antibody specific for MDA. It was subjected to incubation, after which the unbound conjugate was washed off. Next, avidin was conjugated to Horse radish Peroxidase (HRP) and added to each microplate well and incubated. The amount of bound HRP conjugate was taken to be inversely proportional to the concentration of MDA in the sample. Substrate solution was added which caused development of color. The intensity of color that was developed was measured using a microplate reader and the reading was taken to be inversely proportional to the concentration of MDA in the sample.

\section{Sample Collection}

At the end of the 21 days of administration the animals were sacrificed following chloroform inhalation in a closed chamber. Hippocampal tissues were harvested and homogenized in $7.4 \mathrm{PH}$ phosphate buffer solutions for oxidative stress analysis.

\section{Statistical Analysis}

The values recorded in learning and memory tests were analyzed using the Kruskal-Wallis one way analysis of variance on ranks followed by Dunn's multiple comparison test.

The MDA concentrations were analyzed using oneway analysis of variance (ANOVA) followed by Turkey's post hoc test to compare the level of significance between groups using SPSS version 22.Data were expressed as mean \pm standard error of mean $(S E M)$. Values of $(P<0.05)$ were considered significant.

\section{RESULTS}

The results of the novel object recognition testis presented in fig. 1. As shown a significant decrease $(p<0.05)$ in the percentage preference score was observed in the CPF treated group when compared to the control. There was significant improvement in the percentage preference score of the pre-treated (RSV $+\mathrm{CPF}$ ) group when compared to the CPF group. Significant decrease was also observed in the CMC group when compared to the pre-treated group (RSV $+\mathrm{CPF})$. The Vit E group showed improvement in the preference score when compared to the CPF group, though not statistically significant. But the pre-treated (RSV + CPF) group showed higher improvement in the preference score when compared to the Vit $\mathrm{E}$ group.

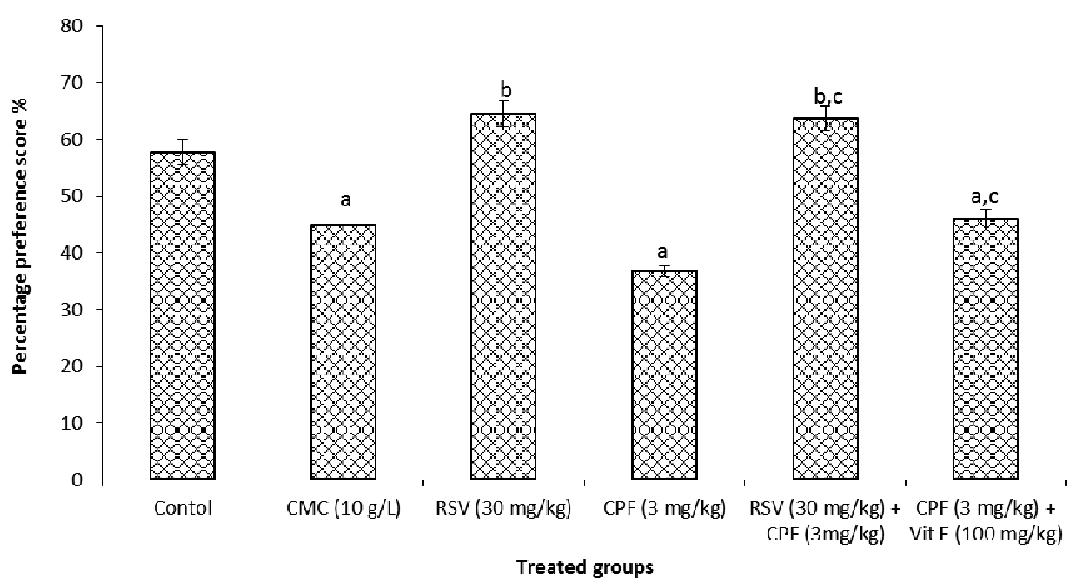

Figure 1: Percentage preference scores (\%) in Swiss albino mice treated with resveratrol, chlorpyrifos and Vitamin E for twenty one (21) days. Control (normal saline, CMC= carboxymethylcellulose, RSV=resveratrol, $\mathrm{CPF}=$ chlorpyrifos, Vit $\mathrm{E}=$ Vitamin $\mathrm{E}, \mathrm{a}, \mathrm{b}, \mathrm{c}=$ significantly different. Result presented as mean $\pm \mathrm{SEM} ; \mathrm{n}=5$

The results of the $\mathrm{Y}$-maze testis presented in fig. 2. As shown the CPF treated group showed a significantly higher alternation score when compared to the control. The pre-treated (RSV + CPF) group showed a significantly lower alternation score when compared to the CPF treated group. A lower alternation score was observed in the Vit $\mathrm{E}$ treated group when compared to the CPF treated group, though not statistically significant. The Vit E treated group also showed a higher alternation score when compared to the RSV pre-treated group. 


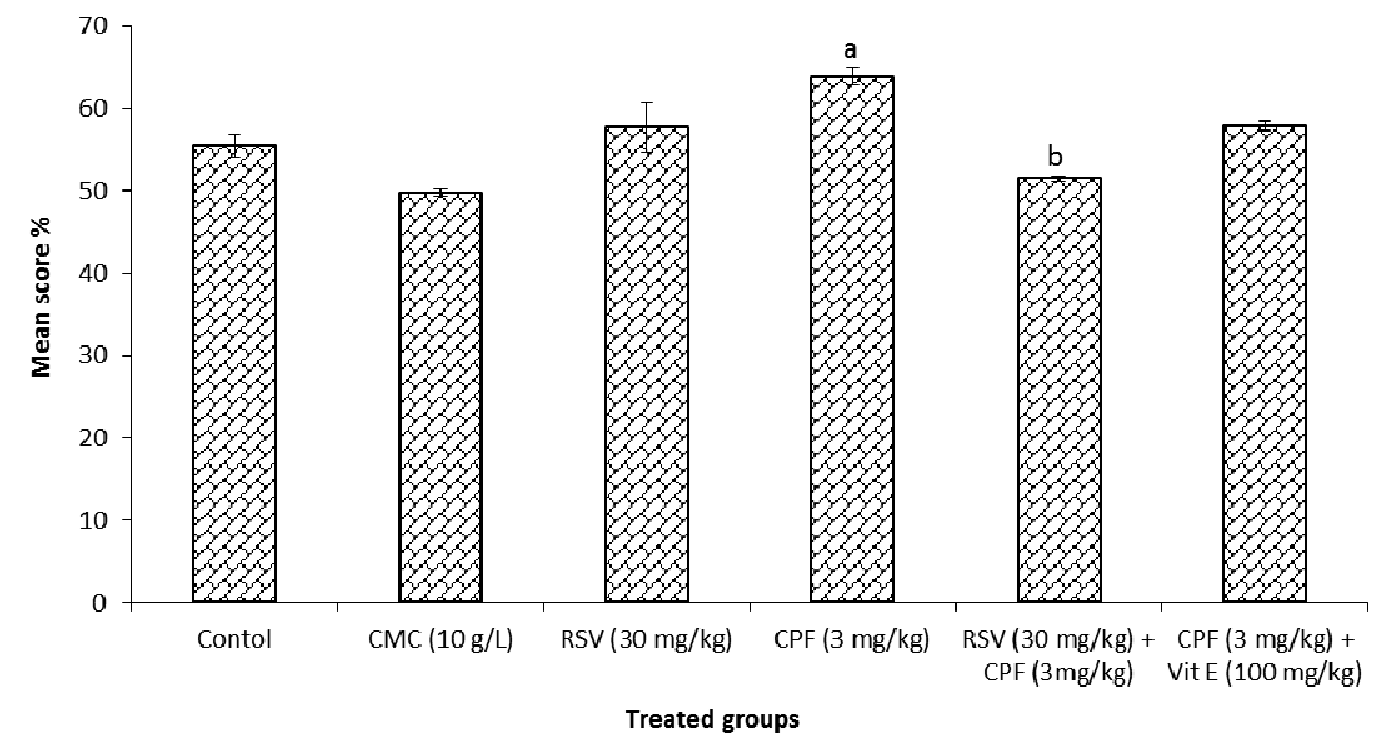

Figure 2: Mean scores (\%) in Swiss albino mice treated with resveratrol, chlorpyrifos and Vitamin E for twenty one (21) days. Superscript $b$ indicates statistical significant difference $(P<0.05)$ when compared to CMC. Result presented as mean $\pm \mathrm{SEM} ; \mathrm{n}=5$.

The results of the malondialdehyde (MDA) activity in the hippocampus of miceis presented in fig. 3. As shown a significant increase in malondialdehyde level in the CPF treated group when compared to the control. There was also a significant increase in malondialdehyde level in the RSV pre-treated group when compared to the control. A significant decrease was observed in malondialdehyde level in the RSV pre-treated group when compared to the CPF treated group. A significant decrease was also observed in the malondialdehyde level of the Vit $\mathrm{E}$ group when compared to the CPF treated group. There was a decrease level of malondialdehyde level in the Vit $\mathrm{E}$ group when compared to the RSV pre-treated group, though not statistically significant.

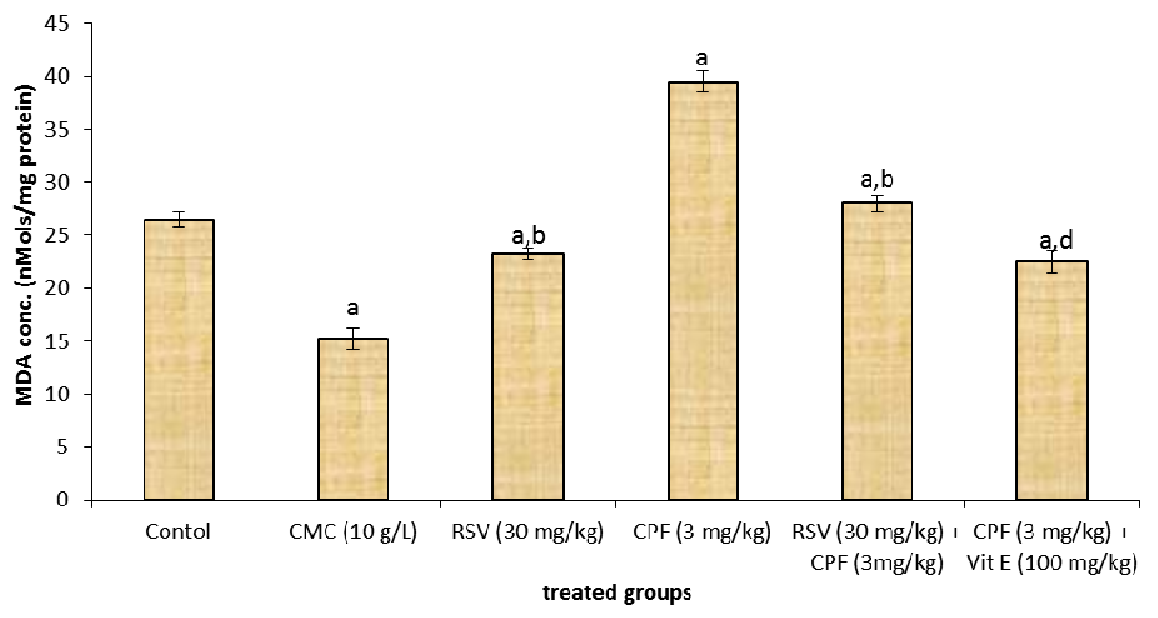

Figure 3: Hippocampal malondialdehyde concentration (nMols/mg) in Swiss albino mice treated with resveratrol, chlorpyrifos and Vitamin E for twenty one (21) days. Superscripts a, b and d indicate statistical significant difference $(P<0.05)$ when compared to Control, CMC and CPF respectively. Result presented as mean \pm SEM; $\mathrm{n}=5$.

\section{DISCUSSION}

This study was designed to evaluate the effect of resveratrol on chlorpyrifos-induced cognitive impairment and lipid peroxidation biomarkers in Swiss albino mice. The Y-maze and the Novel Object Recognition Test (Temporal order task) paradigms were employed to assess the cognitive functions of the mice.
Temporal order task is a method of assessing the recognition memory in rodents. This task comprised two training phases and one test trial. In each training phase, the subjects are allowed to explore two copies of an identical object. Different objects are used for training phases 1 ( $A 1$ and $A 2$ ) and 2 (B1 and $B 2$ ), with a delay between the training phases of $1 \mathrm{~h}$. 
The test trial is given $3 \mathrm{~h}$ after training phase 2 . During the test trial, an objects from training phase 1 (A1) and an objects from training phase 2 (B1) are used. The positions of the objects in the test and the objects used in training phase 1 and 2 are counterbalanced between the animals. If temporal order memory is intact, the subjects will spend more time exploring the object from training 1 (i.e., the object presented less recently) compared with the object from training 2 (i.e., the "new" object).The following parameters are analyzed: the time spent exploring each objects $A 1$ and $A 2$ in the training phase, the time spent exploring each objects $B$ and $A 2$ (object recognition) or objects $A 1 n$ (A1in its new location) and $A 2$ (object location) in the test phase. The data are expressed as the percentage (\%) of time that the animals explore identical objects $(\mathrm{tA} 2 /[\mathrm{tA} 1+\mathrm{tA2}] \times 100)$ during training and the \% of time that the animals explore the novel object (tB/[tB + tA2] $x$ 100) in the retention test (Novel Object Exploration -\% Time) and total exploration time. The time percentage used for the novel object exploration is considered as an index of memory retention (Carlini, 2011).

The results of the Novel Object Recognition test showed significant increase in the preference scores in the group that received resveratrol prior to chlorpyrifos (CPF) administration demonstrating improvement in the recognition memory when compared to the CPF treated group. This shows that administration of resveratrol improved recognition memory.

The $\mathrm{Y}$-maze test is based on the innate preference of animals to explore an arm that has not been previously explored (Drewa, 1998). An alternation is defined as entry into all three arms consecutively (Hughes, 2004), for instance if the animal makes the following arm entries; $A, C, C . A, B, C, A, C, B, A, B, C, A$ in this example, the animal made 13 arm entries 7 of which are correct alternations. The number of maximum spontaneous alternations is then the total number of arms entered minus two, and the percentage alternation is calculated as ((actual alternations /maximum alternations) x 100). A high alternation rate is indicative of sustained spatial working memory as the animals must remember which arm was entered last to not re-enter it. Y-maze function is sensitive to damage in areas concerned with learning and memory functions such as the hippocampus (Hughes, 2004).

The results of Y-maze percentage alternation scores demonstrated a rather decrease in the spartial working memory of the RSV pre-treated mice when compared to the CPF treated mice suggesting that resveratrol does not improve spatial working memory. Lipid peroxidation is often assayed by measuring thiobarbituric acid reactive substances. The end products of lipid peroxidation, such as malondialdehyde assessment, have been widely used to indicate oxidative stress in many studies. Greilberger et al., (2008) used malondialdehyde, carbonyl proteins and albumin-disulphide as oxidative stress parameters to test whether oxidative stress had a primary role in cognitive impairment and Alzheimer's disease. MDA is in many instances the most abundant aldehyde arising from lipid peroxidation (Verma and Srivastava, 2003) and hence is used as an index of oxidative damage in tissues. The increased MDA concentration indicates an ongoing oxidative damage to the brain tissues. The consequence of CPF-induced increased lipoperoxidation in the tissues is biological dysfunctions as the brain is easily susceptible due to its inherent factors (Eren et al., 2007) as a result of interference with their cellular integrities and alterations in their cytostrutural organizations. In contrast, malondialdehyde levels were decreased significantly after treatment with resveratrol in the hippocampus, suggesting that resveratrol could protect mice from chlorpyrifos-induced cognitive impairment through regulating malondialdehyde levels and inhibiting excessive lipid peroxidation.

The present study has shown that chronic CPF exposure causes oxidative changes in the hippocampal tissue. Therefore, we speculate that some of the cognitive deficits that have been previously associated with chronic CPF exposure may be partly due to this oxidative damage. And that supplementation with resveratrol has equally been shown in the present study to mitigate the oxidative damage to the hippocamal tissues and therefore consequently reduced the adverse cognitive dysfunctions outcome associated with CPF exposure.

\section{CONCLUSION}

In conclusion, $30 \mathrm{mg} / \mathrm{kg}$ resveratrol suppressed memory impairment, decreased malondialdehyde levels, in the chlorpyrifos-induced cognitive impairment mice model. The results confirmed the neuroprotective effects of resveratrol on CPF-induced cognitive impairment, and provided novel insights into the neuroprotective effects of resveratrol and its possible therapeutic role in cognitive impairment.

\section{RECOMMENDATIONS}

1. Further studies should be conducted to ascertain the definitive roles play by oxidative injury in causing cognitive dysfunction among CPF users.

2. Awareness campaign should be conducted regularly in the agricultural communities on the dangers and implications of organophosphate pesticides and the use of protective clothing (e.g. face mask, gloves, etc) should be encouraged among both farm and domestic users of chlorpyrifos and other organophosphate pesticides.

3. Further studies should be conducted to ascertain the benefits or otherwise of the long term intake of resveratrol and to check if its beneficial effects are dose-dependent or not.

4. Further studies should be conducted on the effects of long-term intake of resveratrol on other organ-systems of the body to ensure detrimental side effects are avoided.

\section{Contribution of Authors'}

ADAMU BY: Experimental design, concept, neurobehavioral studies, Practical experimentation and manuscript preparation.

AlhassanA W: Experimental design, concept, neurobehavioral studies and manuscript preparation. SALEH MIA: Experimental design, concept, neurobehavioral studies and manuscript preparation. 
YUSUF A:Neurobehavioral studies, Practical experimentation, Statistical analysis and manuscript preparation.

GARKUWA UA: Neurobehavioral studies andtissue preparation

ALIYU M: Neurobehavioral studies

ILIYA BT:Neurobehavioral studies and Practical experimentation

ZUBERU J: Neurobehavioral studies andtissue preparation

LAWAN I: Chemical formulation and supplements standardization

\section{REFERENCES}

Abdollahi, M., Ranjbar, A., Shadnia, S., Nikfar, S. and Rezaie, A. (2004). Pesticides and oxidative stress: a review. Medical Science Monitor, 10 (6).

Ambali, S. F., Abbas, S. O., Shittu, M., Dzenda, T., Kawu, M. U., Salami, S. O. and Ayo, J. O. (2009). Effects of gestational exposure to chlorpyrifos on implantation and neonatal mice. Journal of Cell Animal Biology, 3(4): 050-057.

Ambali, S. F., Makinde, A. O., Shittu, M. and Adeniyi, S. A. (2012). Alleviating effect of Phyllanthus niruri on sensorimotor and cognitive changes induced by subacute chlorpyrifos exposure in Wistar rats.African Journal of Medicine and Molecular Sciences, 2(3): 50-58.

Antunes, M. Biala, G.(2012). The novel object recognition memory: neurobiology, test procedure, and its modifications. Cognitive Process, 13:93-110.

Barker G. R. J., Bird F., Alexander V. and Warburton E. C. (2007) Recognition memory for objects, place, and temporal order: a disconnection analysis of the role of the medial prefrontal cortex and perirhinal cortex. Journal of Neuroscience, 27:2948-2957.

Baxter M. G. (2010) "I've seen it all before": explaining age-related impairments in object recognition. Behavioral Neuroscience, 124:706-709

Carlini, V. P. (2011).The Object Recognition Task: A New Proposal for the Memory Performance Study. Physiology Institute, Medicals Science School, Córdoba National University, CórdobaArgentina, 978-953-307-222-7.

Dercks, W., and Creasy, L. L. (1989a). The significance of stilbenephytoalexins in the Plasmoparaviticola-grapevine interaction. Physiology and Molecular Plant Pathology, 34 189-202.

Drewa, G., Jak.bczyk, M. and Araszkiewicz, A. (1998). Role of free radicals in schizophrenia. Medical Science Monitor, 4(6): 1111-1115.

Eren, I., Naziroglu,M., Demirdas,A. Celik, O. and Uguz, A.C. (2007). Venlafaxine modulates induced oxidative stress in brain and medulla

\section{Conflicts of Interest}

The authors declare that there is no conflict of interests in the publication of this paper.

\section{Acknowledgements}

The authors are grateful to Bauchi State University Gadau, Bauchi State, Nigeria. And Ahmadu Bello University, Zaria, Kaduna State, Nigeria for supporting this project. The authors would also like to thank the staff of the Department of Human Physiology and neurobehavioral laboratory and that of Histology Unit, Anatomy Department, Faculty of Medicine, Ahmadu Bello University, Zaria, Kaduna State, Nigeria.

of rat. Neurochemistry Research, 32: 497505.

Farahat, F. M., Fenske, R. A., Olson, J. R., Garvin, K., Bonner, M. R., Rohlman, D. S., Farahat, T. M., Lein, P. J. and Anger, W. K. (2010). Chlorpyrifos exposures in Egyptian cotton field workers. Neurotoxicology, 31: 297-304.

Fremont, L. (2000). Biological effect of resveratrol. Life Science, 66(8): 663-673.

Gandhi, S. and Abramov, A. Y. (2012). Mechanism of oxidative stress in neurodegeneration. Oxidative Medicine and Cell Longevity, 2012:428010.

Greilberger J, Koidl C, Greilberger M, (2008). Malondialdehyde, carbonyl proteins and albumin-disulphide as useful oxidative markers in mild cognitive impairment and Alzheimer's disease. Free Radicals Research, 42 (7):633-638.

Greilberger J., Koidl C., and Greilberger M., (2008). Malondialdehyde, carbonyl proteins and albumin-disulphide as useful oxidative markers in mild cognitive impairment and Alzheimer's disease. Free Radicals Research, $42(7): 633-638$.

Hughes, R.N. (2004). The value of spontaneous alternation behavior (SAB) as a test of retention in pharmacological investigations of memory. Neuroscience and Behavioral Reviews, 28: 497-505.

Juan, M. E., Vinardell, M. P., and Planas, J. M. (2002). The daily oral administration of high doses of trans-resveratrol to rats for 28 days is not harmful. Journal of Nutrition, 132, 257-260.

Kamel, F. and Hoppin, J. A. (2004). Association of pesticide exposure with neurologic dysfunction and disease. Environmental Health Perspective, 112(9): 950-958.

Kamel, F., Engel, L. S., Gladen, B. C., Hoppin, J. A., Alavanja, M. C. R. and Sandler, S. P. (2007). Neurologic symptoms in licensed pesticide applicators in the agricultural health study. Human and Experimental Toxicology, 26: 243-250.

Kim, D., Nguyen, M. D. and Dobbin, M. M. (2007). SIRT1 deacetylase protects against neurodegeneration in models for Alzheimer's disease and amyotrophic lateral sclerosis. EMBO Journal, 26(13):3169-3179. 
Muhammad, S. M., Magaji, R. A., Mohammed A., Isa, A. S. and Magaji, M. G. (2014). Effect of resveratrol as caloric restriction mimetic and environmental enrichment on neurobehavioural responses in young healthy mice. Advances in Neuroscience, 545170.

Nonomura, S., Kanagawa, H. and Makimoto, L. (1963). A. Chemical constituents of polygonaceous plants. I. Studies on the components of Ko-jo-kon (PolygonumcuspidatumSieb. etZucc.). YakugakuZasshi, 83: 988-990.

Rawat, D. S., Singh, S. P., Sharma, L. D., Ahamad A. H. and Mehta, G. (2003). Residue analysis of some pesticides in poultry egg and mean samples in Garhwal region of Uttaranchal. 22nd Annual Conference of Society for Toxicology, 23-24.

Steenland, K., Dick, R. B., Howell, R. J., Chrislip, D. W., Hines, C. J., Reid, T. M., Lehman, E., Laber, P., Krieg, E. F., and Knott, C. (2000). Neurologic function among termiticide applicators exposed to chlorpyrifos. Environmental Health Perspective, 108 (4): 293-300.
Takaoka, M. J. (1940). Of the phenolic substances of white hellebore (Veratrumgrandiflorum Loe. fil Journal of Faculty of Science, Hokkaido Imperial University 3, 1-16.

Terry Jr., A. V., Gearhart, D. A., Beck Jr., W. D., Truan, J. N., Middlemore, M., Williamson, L. N., Bartlett, M. G., Prendergast, M. A., Sickles, D. W. and Buccafusco, J. J. (2007). Chronic intermittent exposure to chlorpyrifos in rats: Protracted effects on axonal transport, neurotrophin receptors, holinergic markers, and information processing. Journal of Pharmacology Experiments and Therapeutics, 322: 1117-1128.

Verma, R.S. and Srivastava,N. (2003). Effects of chlorpyrifos on thiobarbituric acid reactive substances, scavenging enzymes and glutathione in rats tissues. Indian Journal of Biochemistry and Biophysics, 40: 423428.

Warburton, D. M. (1995). Effect of caffeine on cognition and mood without caffeine abstinence. Psychopharmacology, 119, 6670. 